\title{
A Takagi-Sugeno Fuzzy Rule-Based Model for Soil Moisture Retrieval From SAR Under Soil Roughness Uncertainty
}

\author{
Niko E. C. Verhoest, Bernard De Baets, and Hilde Vernieuwe
}

\begin{abstract}
Radar remote sensing has shown its potential for retrieving soil moisture from bare soil surfaces. Since the backscattering process is also influenced by soil roughness, the characterization of this roughness is crucial for an accurate soil moisture retrieval. However, several field experiments have shown a large variability of the roughness parameters. Describing these parameters by means of possibility distributions allows to account for their uncertainty. Verhoest et al. introduced a retrieval procedure which calculates from these uncertain roughness parameters the possibility distribution of retrieved soil moisture, from which a soil moisture value and uncertainty upon the retrieval are estimated. The main disadvantage of their technique is the high computational demand, which hampers an operational application. In this paper, a fuzzy modeling approach, which is based on fuzzy rules of the Takagi-Sugeno type, is introduced that accurately simulates the soil moisture and the uncertainty upon its retrieved value as obtained by the possibilistic procedure.
\end{abstract}

Index Terms-Fuzzy model, possibility theory, SAR, soil moisture, soil roughness, uncertainty.

\section{INTRODUCTION}

$\mathbf{S}$ OIL roughness is an important factor that, among others, determines the scattering of microwaves at the Earth's surface. Therefore, it is important to accurately know the roughness state in order to invert the backscattered signal into soil moisture. Several studies, however, have shown that soil roughness is very variable, even within one field [2], and its parameterization is not straightforward as, for instance, it depends on the profile length that is used to determine the roughness parameters [2]-[4] or the type of measuring device used [5]. This leads to a large uncertainty upon the roughness parameters, causing an increase of the uncertainty upon the retrieved soil moisture values. Furthermore, when applying Synthetic Aperture Radar (SAR) remote sensing of soil moisture at a large scale in an operational mode, it is impossible to perform soil roughness experiments at each field, and thus,

Manuscript received September 5, 2006; revised January 5, 2007. This work was supported in part by the Belgian Science Policy Office in the frame of the STEREO I (Project SR/00/01) and STEREO II program (Project SR/00/100) and in part by the ESA ITT Project AO/1-4877/05/NL/HE.

N. E. C. Verhoest is with the Laboratory of Hydrology and Water Management, Ghent University, 9000 Ghent, Belgium (e-mail: Niko.Verhoest@ UGent.be).

B. De Baets and H. Vernieuwe are with the Department of Applied Mathematics, Biometrics and Process Control, Ghent University, 9000 Ghent, Belgium.

Digital Object Identifier 10.1109/TGRS.2007.894930 average roughness parameters, which may be assigned to a type of tillage, need to be assumed. Of course, one should be aware that this approach may lead to significant errors on the retrieved soil moisture values.

Satalino et al. [6] investigated the retrieval accuracy when limited soil roughness information is available. They applied artificial neural networks to invert the backscattering coefficient into soil moisture content. In order to train the neural network, Satalino et al. [6] used the traditional Integral Equation Model (IEM) [7], [8] that is valid for a single-scale description of the surface roughness and the multiscale IEM [9] which is based on a band-limited fractal process description of surface roughness. They found that the main source of error in the retrieved volumetric moisture content was caused by the variations in roughness conditions, and that the use of a single- or multiscale roughness description was not critical for inversion studies [10]. Mattia et al. [10] tried to constrain the range of possible retrieval values using a priori information on soil moisture, which was obtained from a simple water balance model, and a priori surface roughness, which was derived from an empirical model. Simulated and experimental results demonstrated that this technique resulted for multipolarized (i.e., HH- and VV-polarization) C-band data in an accuracy of approximately 5 vol.\% for the retrieved soil moisture, provided that sufficient accurate a priori information on surface parameters is available. Soil moisture retrieval from single polarized data is less accurate, but the difference in accuracy with multipolarized data decreases when the uncertainty upon the geophysical parameters increases [10].

Verhoest et al. [1] have demonstrated a procedure which allows to account for the uncertainty upon the roughness parameters. This procedure is based on describing roughness by means of possibility distributions for the root-meansquare (rms) height and correlation length. Through inverting a backscattering model (IEM) for all possible combinations of rms and correlation length, a possibility distribution of soil moisture is obtained, which is then used to calculate the retrieved soil moisture value and the uncertainty thereupon. Although this procedure yields satisfactory results with respect to retrieval uncertainty, Verhoest et al. [1] pointed out that the procedure was computationally very intensive due to the large amount of backscatter model inversions needed. In this paper, it is shown that Takagi-Sugeno fuzzy rule-based models can be used to simulate the retrieval results of the possibilistic procedure, with the advantage of being extremely fast. 
This paper is structured as follows. In Section II, a short overview of the possibilistic retrieval procedure of Verhoest et al. [1] is given, and Takagi-Sugeno fuzzy rulebased modeling is introduced. Section III describes the derivation of the Takagi-Sugeno fuzzy rule-based retrieval model. The results of the model are discussed in Section IV. Finally, conclusions are drawn.

\section{Methodology}

\section{A. Possibilistic Retrieval Procedure}

Verhoest et al. [1] suggested to describe soil roughness parameters by possibility distributions for a certain type of tillage. Their reasoning, herefore, was twofold. First, roughness measurements may lead to a wide range of roughness parameters even within the same field, and therefore, assigning a single roughness parameter set to that specific field may introduce an error. Second, as the basic idea of remote sensing is to make observations without the need of further in situ measurements, one may subdivide one remotely sensed scene in different fields with the same type of tillage, which all have similar, but not exactly the same, soil roughness. The uncertainty that goes with the roughness parameters makes it difficult to determine the correct probability distribution. Yet, describing the roughness parameters by possibility distributions, which are weaker descriptions than the corresponding probability distributions [11], is more appropriate as they can explicitly deal with uncertainty. Possibility theory, which is introduced by Zadeh [12] as an extension to fuzzy set theory, allows to perform reasoning on imprecise or vague concepts and is a mathematical tool that allows to deal with different types of uncertainty.

Verhoest et al. [1], as well as this paper, assume soil roughness to be single scaled, such that roughness can be described by an autocorrelation function (ACF), which, for tillageinduced roughness, corresponds to the exponential functions [2], an rms height $s$ and correlation length $l$. The latter is defined as the spatial lag over which the ACF remains higher than $1 / e$ [13]. For very rough soils, Oh et al. [14] and Davidson et al. [4] found that the Gaussian ACF often better describes the spatial correlation structure. In function of the type of soil roughness and the profile length, smooth and medium rough soils may also be described as a multiscale process [4], [10]. Rougher soils usually can be described by a single-scale ACF. Fortunately, the choice between the single- or multiscale roughness description is not critical for inversion studies [6], [10]. If roughness would be multiscaled, a similar approach as Verhoest et al. [1] can be applied, where other roughness parameters describing the multiscale roughness should be considered, and an appropriate backscatter model should be applied.

The methodology of the possibilistic retrieval procedure is summarized in the flowchart of Fig. 1. First, a possibility distribution is assigned to the type of tillage under consideration. Therefore, a data set of roughness values for one type of tillage is analyzed, and a symmetrical trapezoidal possibility distribution is chosen that is wide enough to ensure that it covers all roughness values for that type of tillage. It has been shown by Dubois et al. [15] that this type of possibility distribution is optimal when roughness parameters are ill known. In the

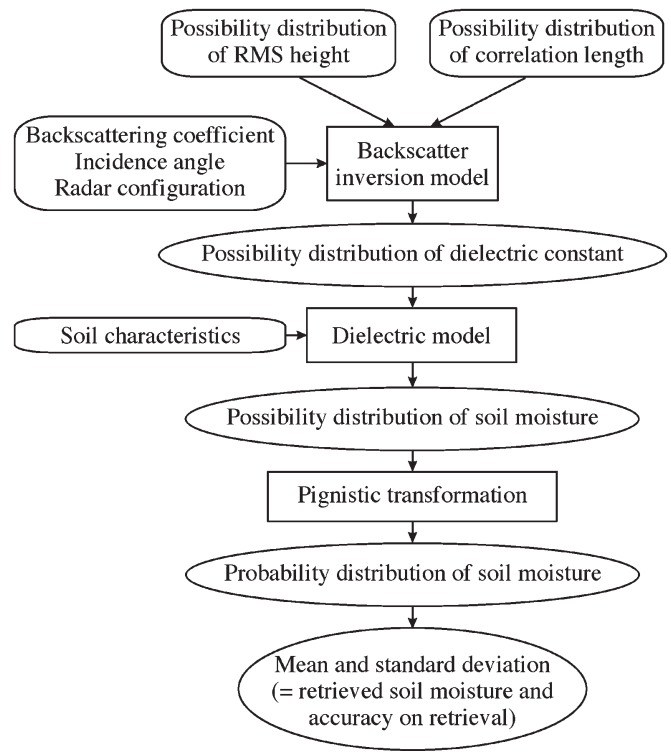

Fig. 1. Flowchart of the possibilistic retrieval procedure of Verhoest et al. [1]. Rounded boxes indicate input, rectangular boxes refer to operations, and ellipses represent output.

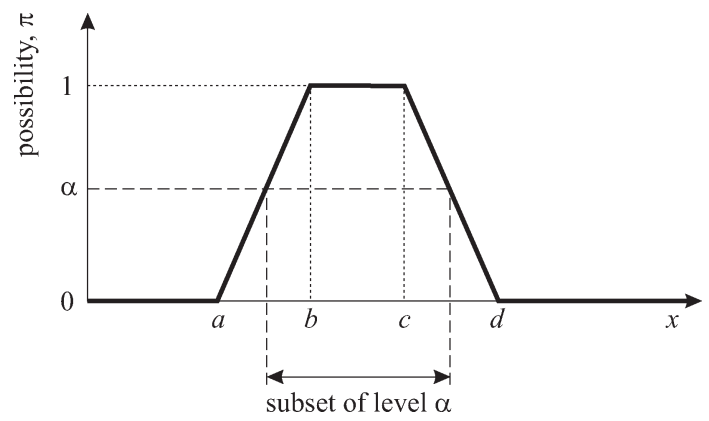

Fig. 2. Example of a possibility distribution of roughness parameter $x$ (i.e., rms height or correlation length).

future work, we will exploit the results of ongoing research on the inference of possibility distributions from the empirical data [16]. Fig. 2 demonstrates such a possibility distribution $\pi$. Roughness values $x$, for which $\pi(x)=1$, are completely possible to occur, whereas when $\pi(x)=0, x$ is an impossible value for the considered type of roughness.

As $s$ and $l$ can be assumed to be separable, we can calculate for the considered type of tillage the possibility of the parameter combination $(x, y)$, with $x$ and $y$, respectively, the rms height, and the correlation length. This is done through the minimum operator

$$
\pi(x, y)=\min \left(\pi_{s}(x), \pi_{l}(y)\right)
$$

where $\pi_{s}$ and $\pi_{l}$ are the possibility distributions of the rms height and the correlation length, respectively. Note that some publications (e.g., [4] and [17]) reveal some dependences between $s$ and $l$. Equation (1) does not explicitly assume any type of dependence and is as such compatible with any dependence assumption. It is important to stress that (1) does not at all reflect independence.

In the next step of the retrieval procedure, for each level $\alpha(\alpha \in[0,1]$, in practice, a step size of 0.05 was chosen), all 
combinations with a possibility larger than $\alpha$ are used to invert the observed backscattering coefficient, using a simplified version of the IEM [18] ( $k s<3$ and $m<0.4$, where $k$ is the wavenumber, and $m$ is the surface slope which equals $s / l$ for an exponentially autocorrelated surface [7], [19]). However, if roughness conditions do not allow its use, other backscattering models should be selected. The obtained dielectric constant is converted into volumetric moisture content using the algorithm of Dobson et al. [20]. If values lower than the residual moisture content are obtained (i.e., 2 vol.\% in the example demonstrated next), then the residual moisture content is retained. If the dielectric mixing model of Dobson et al. [20] results in soil moisture values larger than saturation (i.e., 45 vol.\% for the next test case), then the soil is considered to be saturated. The lowest and highest soil moisture values obtained for all combinations of $s$ and $l$ at a certain $\alpha$ then determine the subset of retrieved soil moisture values at that level $\alpha$. All these subsets determine the possibility distribution of retrieved soil moisture. Finally, the possibility distribution is converted into a probability distribution using the pignistic transformation [21], [22] that tries to preserve the symmetry properties of the possibility distribution into the probability distribution. In order to obtain one specific value for the retrieved soil moisture content, the mean of the obtained probability distribution is calculated. As shown by Verhoest et al. [1], the standard deviation of this probability distribution can be related to the uncertainty upon the retrieval.

Although their methodology was mainly tested using synthetical data, Verhoest et al. [1] applied the possibilistic retrieval algorithm to the European Remote Sensing (ERS) data that are obtained from two sites in Belgium and one site in Italy. However, this required the use of calibrated roughness coefficients, resulting in a root-mean-square error (rmse) between the observed and inverted soil moisture of less than 6 vol.\%.

\section{B. Takagi-Sugeno Fuzzy Rule-Based Model}

Verhoest et al. [1] remarked that the high computational requirement needed when applying the possibilistic retrieval procedure may hamper its application in an operational mode. This shortcoming can be overcome through simulating the inversion results using the data-driven methods such as neural networks or fuzzy modeling. In this paper, we will design a fuzzy rule-based model that is capable of accurately reproducing the results of the possibilistic retrieval procedure, with the major advantage of being very fast when applied.

The Takagi-Sugeno fuzzy rule-based model was first proposed by Takagi and Sugeno [23] and differs from the general Mamdani-Assilian type of fuzzy models [24] by the fact that the consequent part of the fuzzy rules is given by a polynomial function instead of a fuzzy set. For a $p$-dimensional input space, we can define the antecedent fuzzy sets $A_{i}$ as the Cartesian product $A_{i}=A_{1, i_{1}} \times \cdots \times A_{p, i_{p}}$, with $i_{1} \in$ $\left\{1, \ldots, n_{1}\right\}, \ldots, i_{p} \in\left\{1, \ldots, n_{p}\right\}$, and $n_{1}, n_{2}, \ldots, n_{p}$ the number of fuzzy sets each input variable is partitioned into. A membership function is then defined as the mathematical function which defines the degree of the membership of an element in a fuzzy set.
For a first-order Takagi-Sugeno model, we then obtain the $n$ fuzzy rules of the following form

$$
\begin{aligned}
R_{i}: & \operatorname{IF}\left(X_{1}, \ldots, X_{p}\right) \text { is } A_{i} \\
& \text { THEN } Y=a_{1, i} X_{1}+a_{2, i} X_{2}+\cdots+a_{p, i} X_{p}+b_{i}
\end{aligned}
$$

where $a_{1, i}, \ldots, a_{p, i}$, and $b_{i}$ are the parameters of the consequent part of rule $R_{i}$, and $n$ is dependent on the type of identification method (see further). For an input vector $\mathbf{x}=$ $\left(x_{1}, \ldots, x_{p}\right), A_{i}(\mathbf{x})$ is usually calculated as

$$
A_{i}(\mathbf{x})=A_{1, i_{1}}\left(x_{1}\right) \cdot A_{2, i_{2}}\left(x_{2}\right) \cdots A_{p, i_{p}}\left(x_{p}\right) .
$$

The resulting output value $y$ is then computed by weighing the individual rule outputs

$$
y=\frac{\sum_{i=1}^{n} w_{i}(\mathbf{x})\left(a_{1, i} x_{1}+\cdots+a_{p, i} x_{p}+b_{i}\right)}{\sum_{i=1}^{n} w_{i}(\mathbf{x})}
$$

where $w_{i}(\mathbf{x})$ is the degree of fulfillment of rule $i$, which, for the above type of rules, is given by the membership degree $A_{i}(\mathbf{x})$. Through this weighted average, the first-order Takagi-Sugeno model is able to approximate a nonlinear function based on a set of linear functions.

For the construction of a Takagi-Sugeno model, different identification methods can be used. If expert knowledge is available, an expert can define the fuzzy sets of the antecedent variables through their membership function, using prior knowledge and experience. Yet, such human interaction in the process may lead to results that are not repeatable by others or for other data sets. However, if expert knowledge is not available, data-driven identification techniques, such as fuzzy clustering algorithms or grid partitioning, can be applied. Such an approach has the major advantage of being repeatable by different persons and is, therefore, being advised as the technique to be used. Fuzzy clustering algorithms subdivide the input-output space in a number of clusters, each corresponding to a fuzzy rule. Different techniques are available to perform the clustering. The fuzzy $c$-means and the Gustafson-Kessel clustering algorithm [25] are among the most popular, while other algorithms, such as the Gath-Geva clustering algorithm [26], have shown, for a hydrological case study, to perform better [27].

In this paper, grid partitioning will be performed. Initially, the domain of the antecedent variables is subdivided into equidistant and identically shaped membership functions. Fig. 3 shows a possible fuzzy partitioning of the problem considered in this paper: the antecedent variables backscattering coefficient and incidence angle are partitioned into four and three linguistic terms, respectively, and modeled by means of triangular membership functions. Alternative shapes, such as trapezoidal or bell-shaped membership functions, can be chosen. Using the available input-output data (see Section III), the locations of the membership functions can be optimized. The rule base is established in such a way that all combinations of antecedent fuzzy sets are covered, i.e., $n=\prod_{i=1}^{p} n_{i}$ fuzzy rules that have to be optimized, where $p$ is the number of antecedent variables, and $n_{i}$ is the number of membership functions of variable $i$ (e.g., for Fig. 3, $4 \times 3=12$ rules will be obtained). A major 


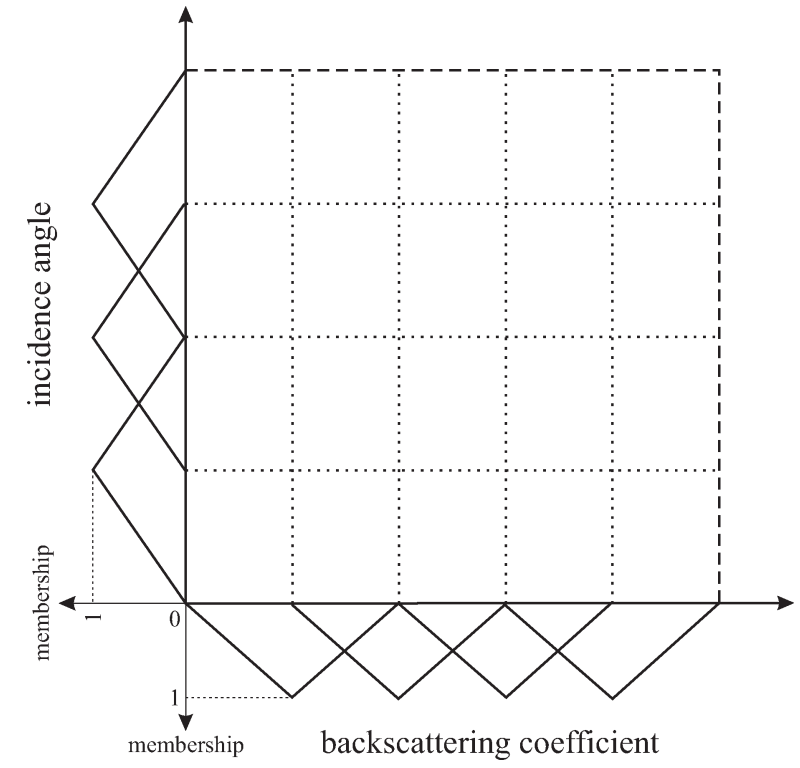

Fig. 3. Example of a grid partitioning of the input domain, which is defined by the backscattering coefficient and the incidence angle in four and three triangular membership functions, respectively.

drawback of this technique is that the membership functions for every variable are constructed independently of each other, causing that the relationship between the variables is neglected. Another disadvantage lies in the fact that since all combinations of antecedent membership functions need to be covered into rules, adding an additional membership function increases the model complexity and makes the identification process more difficult. The number of membership functions chosen should be a tradeoff between the model complexity and the model accuracy. In order to remain linguistically interpretable, the maximum number of membership functions per input variable should not exceed seven [28].

\section{TAKAGI-Sugeno Retrieval Model}

\section{A. Data Set}

In order to derive a fast alternative to the possibilistic retrieval algorithm, i.e., the Takagi-Sugeno retrieval model, a data set was constructed based on the possibilistic retrieval procedure. Therefore, 10000 random combinations of radar backscattering coefficient and incidence angle were inverted for a given roughness description (see Table I) using the possibilistic approach, yielding the retrieved soil moisture as well as the estimated uncertainty upon the retrieval. As will be mentioned later, this data set will be subdivided in data sets for training, checking, and validating of the Takagi-Sugeno models. The roughness possibility distributions of the first class are based on the roughness measurements that are performed with $25-\mathrm{m}$ long laser-derived roughness profiles of rotary tilled soils [2]. The parameters of the trapezoidal possibilities are chosen subjectively, trying to fit the observations. Additional research is needed to estimate the possibility distributions on histograms that are constructed on a small data sets. In order to do so, we will exploit the results of ongoing research on the inference of possibility distributions from the empirical data [16]. However,
TABLE I

DEFINITION OF THE Possibility Distributions of THE RMS HEIGHT AND THE CORRELATION LENGTH FOR THE TWO ROUGHNESS Classes CORRESPONDING TO A ROTARY TILLED FIELD. The PARAmeters $a, b, c$, AND $d$ ARE DeFined IN Fig. 2

\begin{tabular}{ccccc}
\hline & \multicolumn{4}{c}{ RMS height (cm) } \\
roughness class & $a$ & $b$ & $c$ & $d$ \\
\hline class 1 & 0.4 & 0.7 & 1.0 & 1.3 \\
class 2 & 0.7 & 0.8 & 0.9 & 1.0 \\
\hline \multicolumn{5}{c}{ correlation length (cm) } \\
roughness class & $a$ & $b$ & $c$ & $d$ \\
\hline class 1 & 20.0 & 30.0 & 50.0 & 60.0 \\
class 2 & 30.0 & 35.0 & 45.0 & 50.0 \\
\hline
\end{tabular}

the definition of the possibility distributions will not influence the results obtained in this paper. For the second class, a lower uncertainty upon the roughness parameters is given in order to assess the impact of the uncertainty upon the roughness parameters. The random backscattering coefficient was drawn from a uniform distribution extending over a range that was defined by the minimum and maximum backscattering coefficients that were obtained with IEM for all possible soil moisture values and roughness parameters for the considered type of tillage and incidence angles. For the experiment described in this paper, the ERS satellite configuration was adopted [i.e., C-band $(5.3 \mathrm{GHz}) \mathrm{VV}$-polarization]. Due to local topography, the local incidence angle will vary. For the data sets generated, a range of $\left[15^{\circ}, 30^{\circ}\right]$ was chosen, from which the random incidence angles were drawn uniformly.

Fig. 4 displays these data sets, where on the left-hand side, the retrieved soil moisture values are given, while the righthand side demonstrates the estimated uncertainty as predicted by the possibilistic retrieval procedure. With respect to the retrieved value, generally, an overestimation is made for the intermediate soil moisture values, whereas underestimations are observed at higher moisture contents. The extent of these over- and underestimations increases when the uncertainty of the roughness classes increases (i.e., when the possibility distributions become wider). For a detailed discussion on the retrieval results of the possibilistic procedure, we refer to Verhoest et al. [1].

\section{B. Performance Indexes}

The accuracy of the identified Takagi-Sugeno models is evaluated through using the three performance indexes. These are the rmse, the mean absolute error (MAE), and the bias (BIAS) given, respectively, by

$$
\begin{aligned}
\text { rmse } & =\sqrt{\frac{1}{N} \sum_{k=1}^{N}\left(y(k)-y_{\mathrm{m}}(k)\right)^{2}} \\
\mathrm{MAE} & =\frac{1}{N} \sum_{k=1}^{N}\left|y(k)-y_{\mathrm{m}}(k)\right| \\
\text { BIAS } & =\frac{1}{N} \sum_{k=1}^{N}\left(y(k)-y_{\mathrm{m}}(k)\right)
\end{aligned}
$$




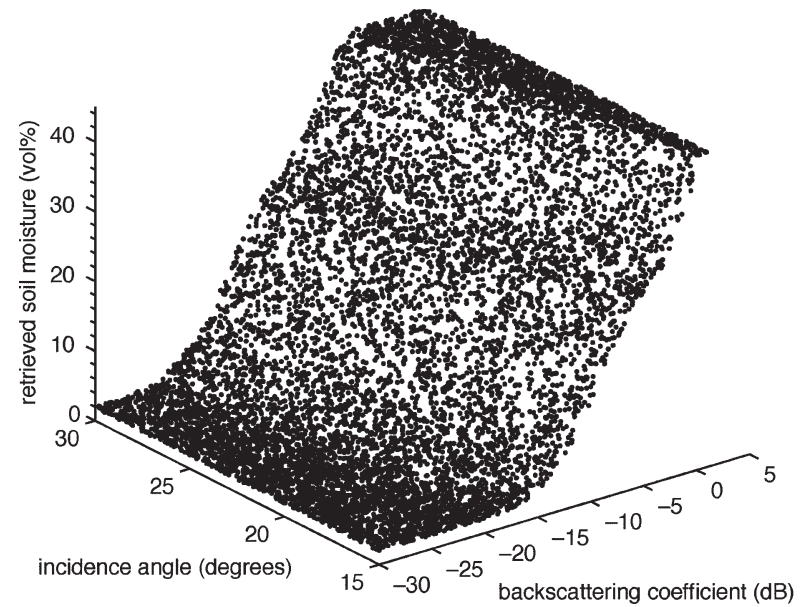

(a)

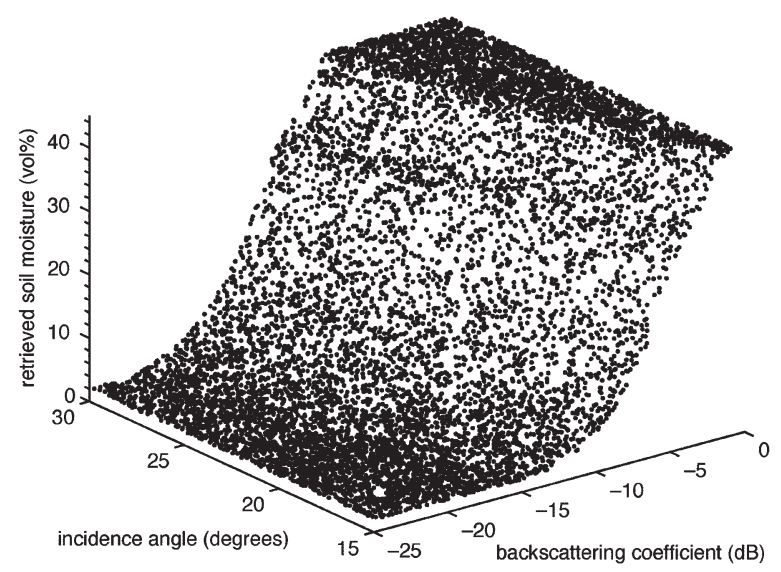

(c)

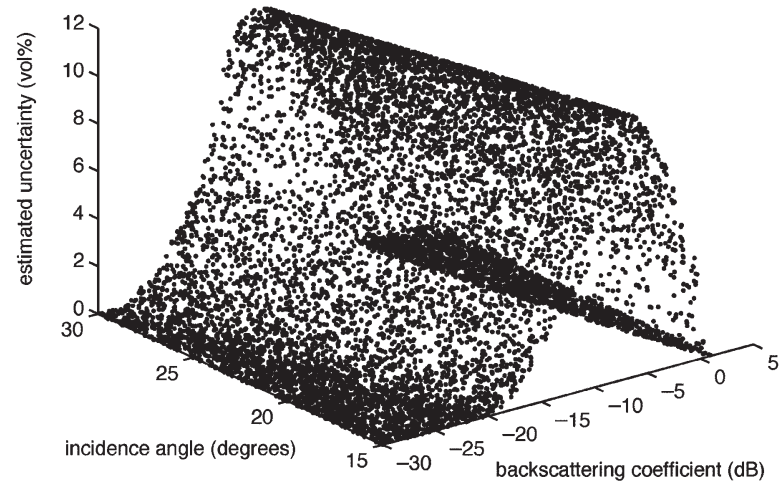

(b)

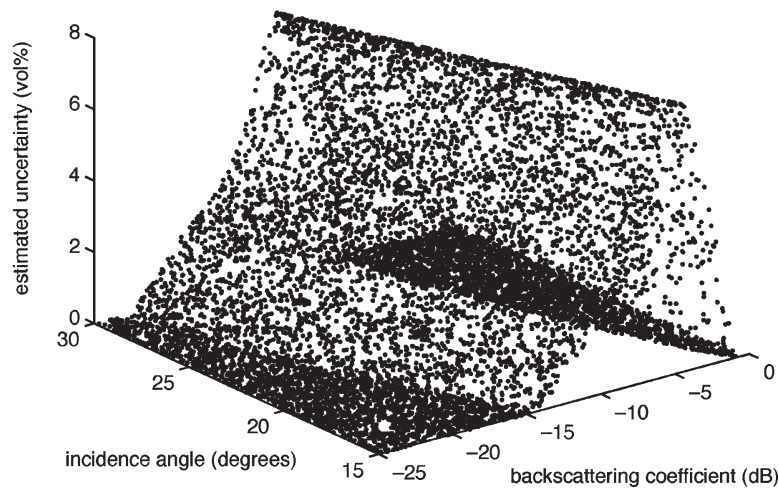

(d)

Fig. 4. Data sets used for the identification and validation of the Takagi-Sugeno models. (a) and (b) Roughness class 1. (c) and (d) Roughness class 2. (a) and (c) Retrieved soil moisture. (b) and (d) Estimated uncertainty as a function of backscattering coefficient and incidence angle.

where $N$ is the number of data points, and $y_{\mathrm{m}}$ and $y$ are the modeled and observed soil moisture, respectively. The optimal values of these performance indexes are zero, indicating a perfect match of the model.

\section{Identification of the Takagi-Sugeno Model}

Since we have no a priori knowledge on the number of membership functions to be used, we varied the number of membership functions for each of the antecedent variables (backscattering coefficient and incidence angle) between three and seven. The identification of the model was based on the adaptive network-based fuzzy inference system (ANFIS) [29], which represents the fuzzy model as a multilayer feedforward neural network. In order to employ ANFIS, the $p$ symmetrical triangular membership functions are constructed which overlap at degree 0.5 with their neighboring membership function (i.e., Ruspini partition [30]), such that the complete input space is covered with membership functions. Then, the parameters of the membership functions are adjusted, and the parameters of the consequent part of the different rules are determined in a learning process through a hybrid method that consists of a backpropagation to estimate the parameters of the antecedent membership functions and a least squares estimation method to identify the parameters of the consequent part.

From the data set, 5000 points were randomly chosen to train the neural network; 2000 other points were used as checking data to prevent overfitting of the model to the training data set. The identified Takagi-Sugeno model corresponds to the model with the lowest rmse on the checking data. As it is possible that the retained model generates impossible soil moisture values (i.e., higher than saturation or lower than residual moisture content), an additional constraint is set that maximizes (minimizes) the Takagi-Sugeno derived soil moisture to the saturated (residual) moisture content. When negative uncertainties are obtained, which are merely an artefact of the Takagi-Sugeno model, and the model is unable to perfectly simulate the sharp transition in the surface when an uncertainty of $0 \mathrm{vol} \%$ is reached, the value was set to zero. Finally, the remaining 3000 points were used for validation.

Tables II and III give the best and worst performance indexes obtained for the Takagi-Sugeno models for the retrieved soil moisture and the estimated uncertainty, respectively, upon the retrieval for all combinations of numbers of membership functions per antecedent variable. Also indicated is the combination at which these optimal values are obtained. Generally, it is 
TABLE II

Best And Worst Performance IndeXes ObTained For the TAKAgi-Sugeno Models of the Retrieved SoIL Moisture When the Number of Membership Functions Per Antecedent Variable Was Varied Between Three and SeVen. Also Given Between Brackets $\left(n_{\mathrm{B}} \times n_{\mathrm{I}}\right)$ Is the Number of Membership Functions for the BacksCattering COEFFICIENT $n_{\mathrm{B}}$ AND INCIDENCE ANGLE $n_{\mathrm{I}}$ FOR WHICH THE INDEX IS OBTAINED

\begin{tabular}{lcccc}
\hline & \multicolumn{2}{c}{ roughness class 1} & \multicolumn{2}{c}{ roughness class 2} \\
& \multicolumn{1}{c}{ best model } & worst model & best model & worst model \\
\hline identification data set & & & & \\
RMSE (vol\%) & $0.090(7 \times 6)$ & $0.635(3 \times 3)$ & $0.172(7 \times 7)$ & $0.472(3 \times 3)$ \\
MAE (vol\%) & $0.055(7 \times 7)$ & $0.422(3 \times 3)$ & $0.096(7 \times 7)$ & $0.298(3 \times 3)$ \\
BIAS (vol\%) & $-1.78 \mathrm{E}-04(4 \times 6)$ & $0.018(4 \times 3)$ & $0.002(4 \times 6)$ & $0.027(3 \times 3)$ \\
\hline validation data set & & & & \\
RMSE (vol\%) & $0.089(7 \times 7)$ & $0.628(3 \times 3)$ & $0.126(7 \times 7)$ & $0.382(3 \times 3)$ \\
MAE (vol\%) & $0.055(7 \times 7)$ & $0.420(3 \times 3)$ & $0.105(7 \times 7)$ & $0.297(3 \times 3)$ \\
BIAS (vol\%) & $-6.75 \mathrm{E}-04(3 \times 5)$ & $0.010(4 \times 3)$ & $0.002(4 \times 7)$ & $0.028(3 \times 3)$ \\
\hline
\end{tabular}

TABLE III

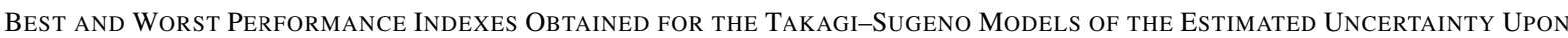
the Retrieval of Soil Moisture When the Number of Membership Functions Per Antecedent Variable Was Varied Between Three and Seven. Also Given Between Brackets $\left(n_{\mathrm{B}} \times n_{\mathrm{I}}\right)$ Is the Number of Membership FUNCTIONS FOR THE BACKSCATTERING COEFFICIENT $n_{\mathrm{B}}$ AND INCIDENCE ANGLE $n_{\mathrm{I}}$ FOR WHICH THE INDEX IS OBTAINED

\begin{tabular}{lcccc}
\hline & \multicolumn{2}{c}{ roughness class 1} & \multicolumn{2}{c}{ roughness class 2} \\
& best model & worst model & best model & worst model \\
\hline identification data set & & & \\
RMSE (vol\%) & $0.140(7 \times 4)$ & $0.463(3 \times 4)$ & $0.118(7 \times 7)$ & $0.377(3 \times 3)$ \\
MAE (vol\%) & $0.079(7 \times 7)$ & $0.314(3 \times 4)$ & $0.067(7 \times 6)$ & $0.230(3 \times 3)$ \\
BIAS (vol\%) & $-0.012(7 \times 5)$ & $-0.063(3 \times 3)$ & $-0.009(7 \times 7)$ & $-0.047(3 \times 3)$ \\
\hline validation data set & & & \\
RMSE (vol\%) & $0.152(7 \times 4)$ & $0.457(3 \times 4)$ & $0.126(7 \times 7)$ & $0.382(3 \times 3)$ \\
MAE (vol\%) & $0.086(7 \times 4)$ & $0.309(3 \times 7)$ & $0.070(7 \times 6)$ & $0.229(3 \times 3)$ \\
BIAS (vol\%) & $-0.012(7 \times 7)$ & $-0.067(3 \times 3)$ & $-0.009(7 \times 7)$ & $-0.048(3 \times 3)$ \\
\hline
\end{tabular}

found that the more membership functions used per antecedent variable, the better the performance index becomes. As can be observed from these tables, the performance indexes for whatever combination of number of membership functions are low (i.e., less than 1 vol.\%, even for the worst case), and therefore, optimizing for the number of membership functions per antecedent variable does not result in models with a significant better performance.

When comparing the different performance indexes on the validation data set with those on the training data set, similar results are found. This reveals that no overfitting of the different models was obtained. Overall, we find that, for all models, the rmse made is less than 0.2 vol.\%, which can be regarded as negligible given the fact that the rmse of the retrieval procedure is approximately 6 vol.\% [1].

\section{RESUlTS}

\section{A. Discussion of the Obtained Takagi-Sugeno Models}

Figs. 5 and 6 display the resulting membership functions or fuzzy sets that are obtained for the retrieved soil moisture for Takagi-Sugeno models with three (top figures), five (middle figures), and seven (bottom figures) membership functions for each of the antecedent variables for the first and second roughness class, respectively. Similar graphs can be made for the Takagi-Sugeno models that simulate the estimated uncertainty. In order to have a good identification, a considerable overlap should exist between adjacent fuzzy sets in order to assure that all points of the input domain are well described by at least one fuzzy set. Furthermore, to be linguistically interpretable, fuzzy sets should not be similar and highly overlapping; redundant membership functions should, therefore, be merged to one fuzzy set [31]. For the different Takagi-Sugeno models that have been identified, a good identification was obtained. However, one should be aware that the models obtained can only be applied in the specific cases for which they were developed. When other roughness conditions or sensor configurations apply, the previously described procedure for identifying the Takagi-Sugeno models needs to be repeated.

As was mentioned before, the overall error made by the fuzzy models is small. However, it is interesting to study the error surface of the Takagi-Sugeno modeled output. Figs. 7 and 8 display the error surfaces together with their probability density of the first and second roughness class, respectively. These surfaces are obtained for the Takagi-Sugeno models with seven membership functions for the backscattering coefficient as well as the incidence angle. As can be seen from the probability density, the errors are not biased (average error is close to zero, cf. the small values of BIAS in Tables II and III), and only a small spread of errors is obtained (cf. the small rmse and MAE values in Tables II and III). The largest errors made by the Takagi-Sugeno models of retrieved soil moisture as well as the estimated uncertainty are found in the transition zone between the nearly saturated and saturated soil moisture. This 

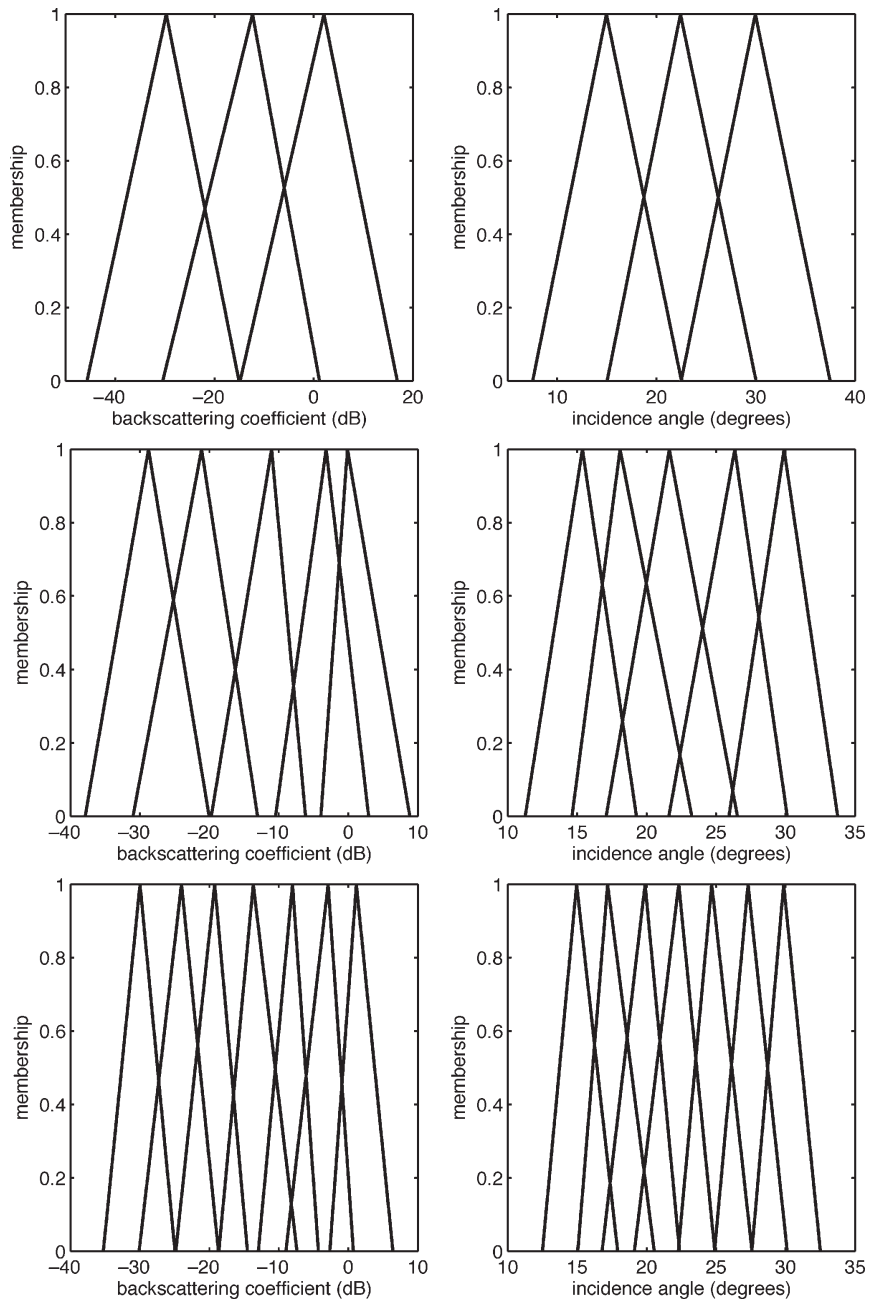

Fig. 5. Membership functions for retrieved soil moisture for roughness class 1.

is mainly caused by the sudden change in slope in the surface once saturation is reached.

\section{B. Comparison of Possibilistic and Takagi-Sugeno Retrieval}

To further validate the Takagi-Sugeno retrieval model, a comparison was set up where the two techniques were applied to 100 synthetical data points. For this experiment, for each class, 100 backscatter values were generated with IEM, using random values for soil moisture (uniformly distributed in the interval [2 vol. \%, $45 \mathrm{vol} . \%]$ and further referred to as observed soil moisture) and incidence angle (uniformly distributed in the interval $\left.\left[15^{\circ}, 30^{\circ}\right]\right)$. The roughness parameters applied are distributed according to the probability distribution derived from the roughness possibility distributions using the pignistic transformation. The obtained backscattering coefficients with corresponding incidence angle were then inverted to soil moisture and estimated uncertainty using both the possibilistic retrieval procedure and the Takagi-Sugeno models, and the CPU time needed was determined. Fig. 9 shows the retrieved soil moisture values for both methods versus the observed soil moisture. As can be seen from this graph, both models generate similar results (rmse on the differences between both techniques, which is calculated over the 100 points equals 0.10
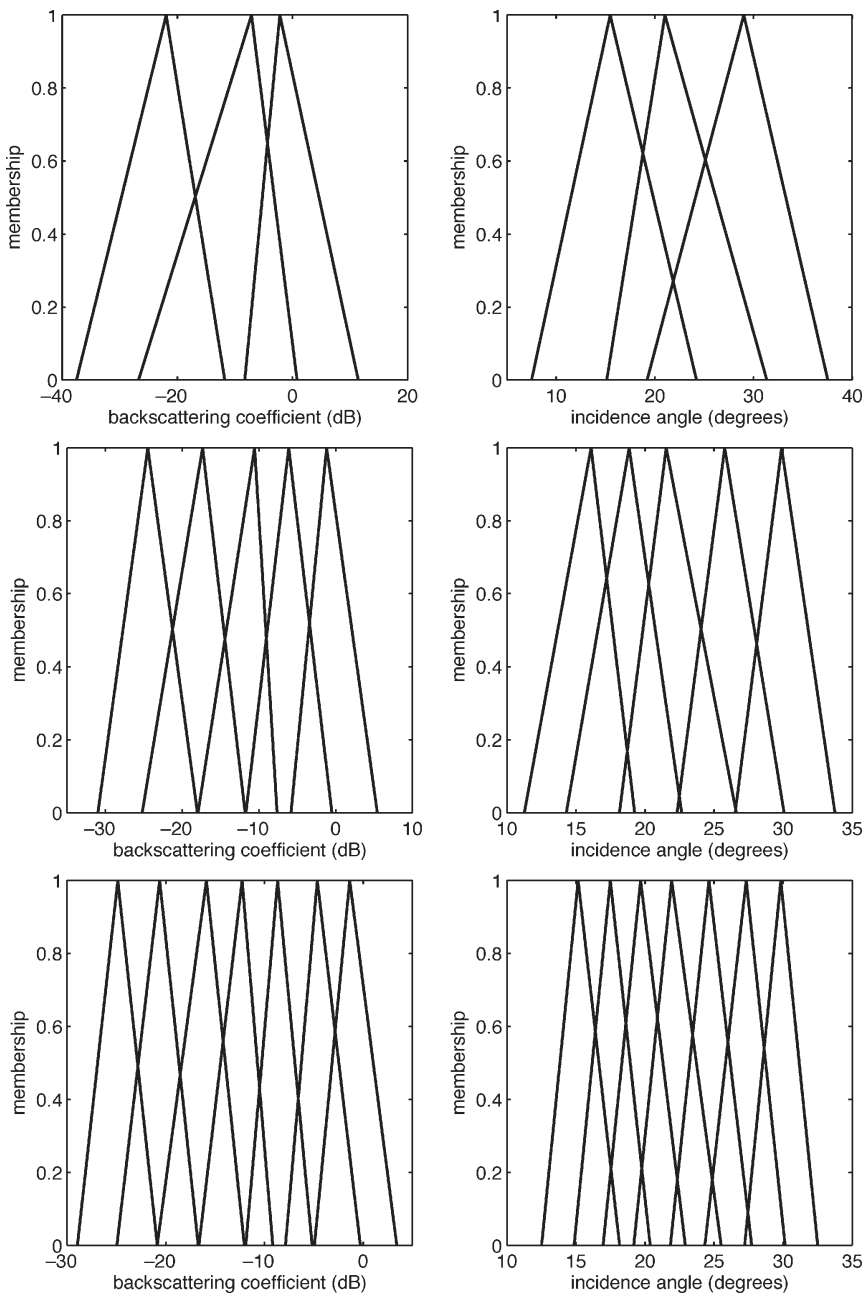

Fig. 6. Membership functions for retrieved soil moisture for roughness class 2 .

and 0.27 vol.\% for roughness classes 1 and 2, respectively). Fig. 10 shows that the uncertainty upon the retrieved soil moisture value as predicted by both techniques is modeled similarly (with an rmse on the differences between the possibilistic and Takagi-Sugeno approach of 0.11 and 0.16 vol.\% for classes 1 and 2, respectively).

As the rmse values on the differences between the possibilistic and the Takagi-Sugeno results are very small compared to the rmse made on the retrieved soil moisture value based on the possibilistic approach, similar rmse values are expected for the Takagi-Sugeno retrieved soil moisture. Fig. 9 demonstrates that soil moisture prediction between both methods is comparable, which is reflected in the fact that the difference in rmse on the soil moisture estimation for both models is less than 0.02 vol. $\%$ (i.e., for class 1, rmse values are 8.528 and $8.530 \mathrm{vol} . \%$ for the possibilistic and Takagi-Sugeno retrieval, respectively; for class 2, values of 4.714 and 4.703 vol.\% are obtained, respectively). With respect to soil moisture, such small difference can be considered as negligible, from which one can conclude that both models retrieve soil moisture with the same accuracy. The above experiment shows that if Takagi-Sugeno retrieval models would be identified for experimental data, similar retrieval results as obtained with the possibilistic retrieval algorithm will be obtained. 


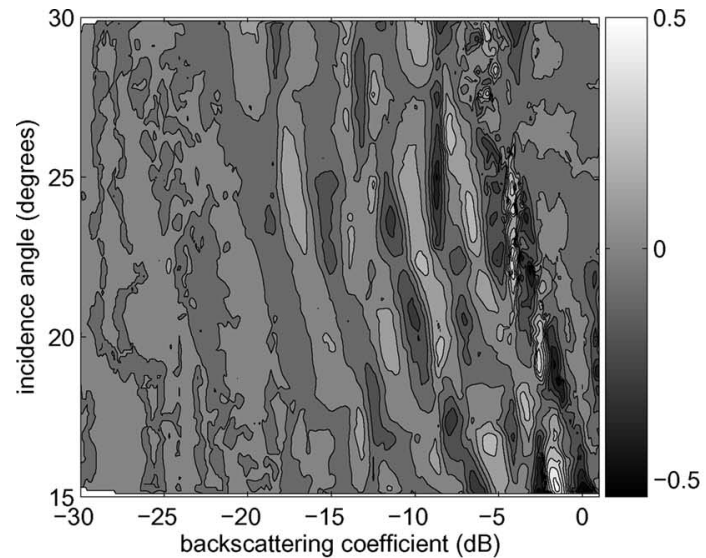

(a)

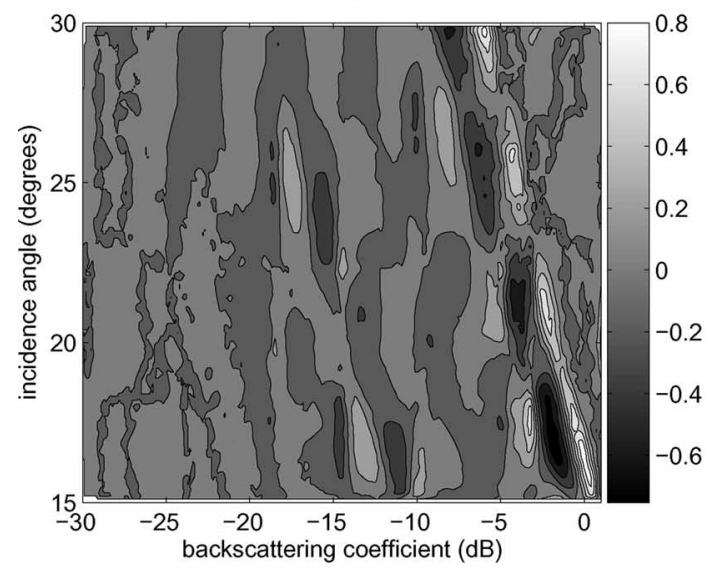

(c)

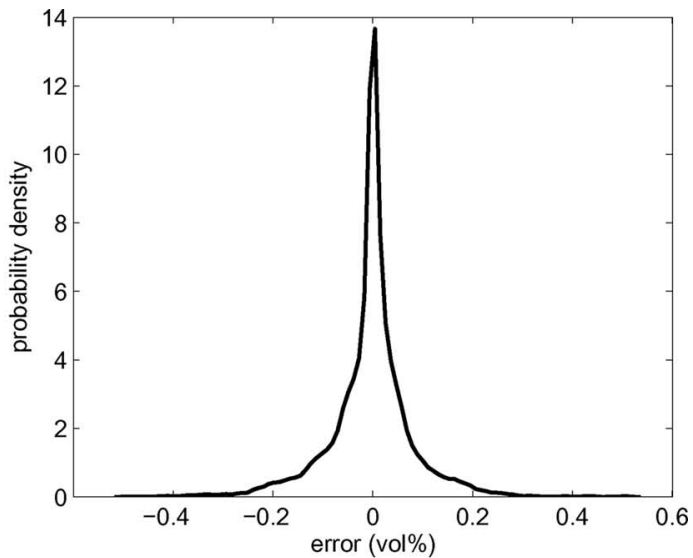

(b)

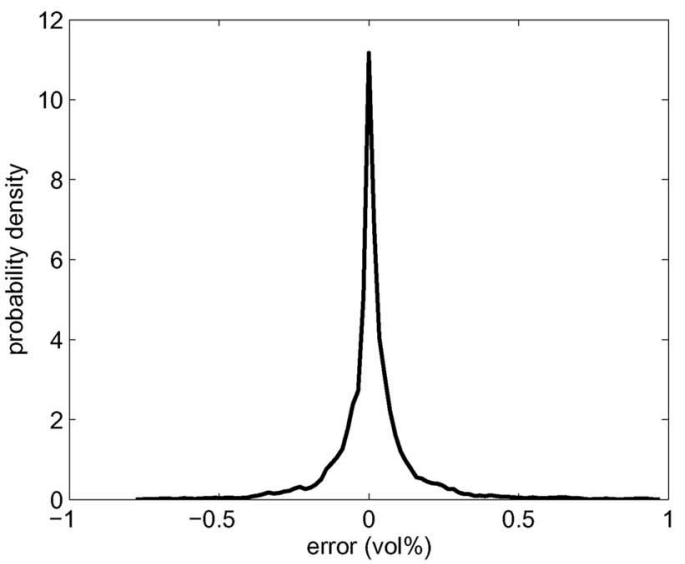

(d)

Fig. 7. Error surface and probability distribution of the error made with the Takagi-Sugeno model for the class 1 roughness. (a) and (b) Retrieved soil moisture. (c) and (d) Estimated uncertainty.

\section{Comparison of Runtime}

The main objective of developing the Takagi-Sugeno fuzzy rule-based model was to overcome the high computational effort and, consequently, the runtime needed by the original possibilistic retrieval procedure. Therefore, a comparison of the runtime that is needed for both methods on the 100 randomly chosen data points was made.

The possibilistic retrieval procedure needed 3934 and $4584 \mathrm{~s}$ CPU time on a pentium 2-GHz Intel processor for the first and second roughness class, respectively. The Takagi-Sugeno model required $0.0005 \mathrm{~s}$ in both cases. These values clearly demonstrate the benefit of using the Takagi-Sugeno approach above the possibilistic retrieval procedure without loss of accuracy. Yet, one should be aware of the high computational load for the construction of the data set (10000 points require more than $100 \mathrm{~h}$ of CPU time) and the identification (CPU time depends on the number of membership functions per antecedent variable, ranging from $1 \mathrm{~min}$ for three membership functions for each input variable to 22 min for seven membership functions for each antecedent variable for the identification of one Takagi-Sugeno model). This computational effort should only be made once, and if the retrieval model needs to be applied in an operational mode, this exercise is worth the effort.

\section{Conclusion}

Takagi-Sugeno fuzzy rule-based models have been identified that they are able to retrieve the soil moisture and the uncertainty upon the retrieval from SAR backscattering coefficients. The possibilistic retrieval procedure (developed by Verhoest et al. [1]), which allows to account for soil roughness uncertainty, was applied to generate the training data needed to identify the Takagi-Sugeno models. This requires an a priori knowledge on the tillage state which may be derived from crop calendars as this information allows to reveal the tillage state of the fields. Then, for each tillage state, possibility distributions can be deduced from roughness data or through calibration as demonstrated by Verhoest et al. [1]. Future research will address this problem in which a recently developed technique for inferring possibility distributions from the empirical data [16] will be applied. Grid partitioning was used, and the number of membership functions was varied between three and seven. It was found that, even with three membership functions for both antecedent variables (backscattering coefficient and incidence angle), accurate results were obtained with the fuzzy rule-based models. Increasing the number of fuzzy sets per antecedent variable generally increased the performance of the models. 


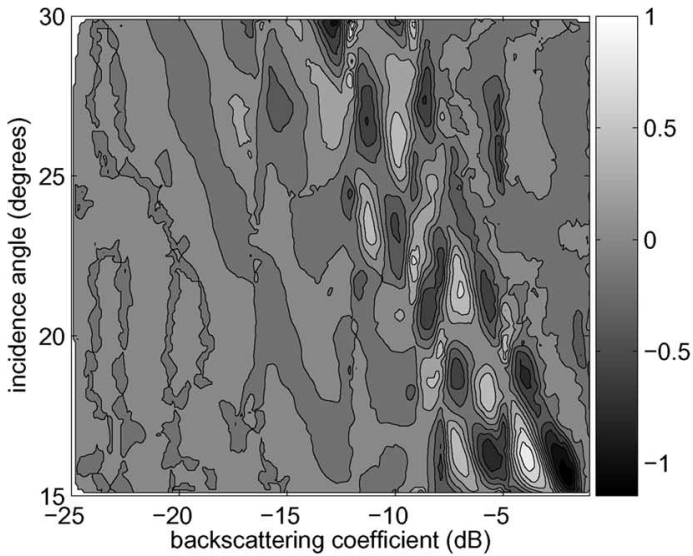

(a)

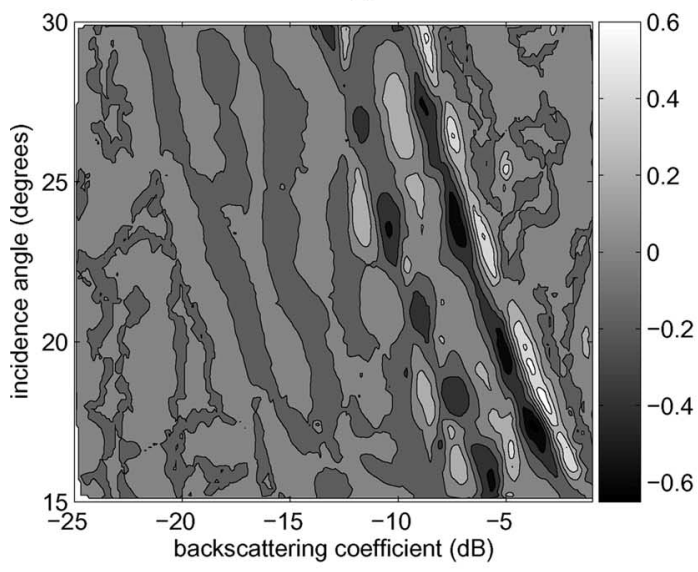

(c)

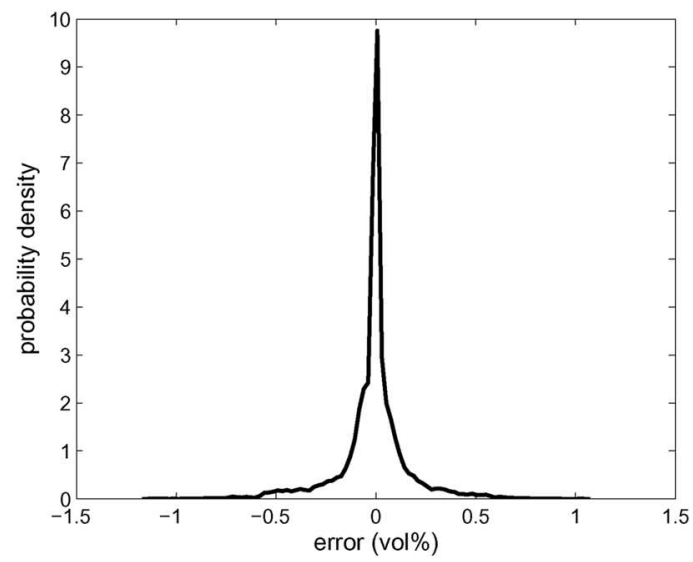

(b)

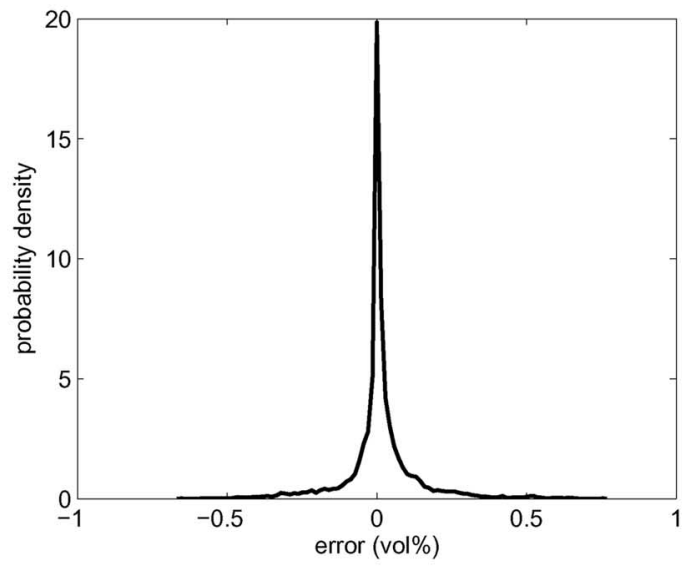

(d)

Fig. 8. Error surface and probability distribution of the error made with the Takagi-Sugeno model for the class 2 roughness. (a) and (b) Retrieved soil moisture. (c) and (d) Estimated uncertainty.

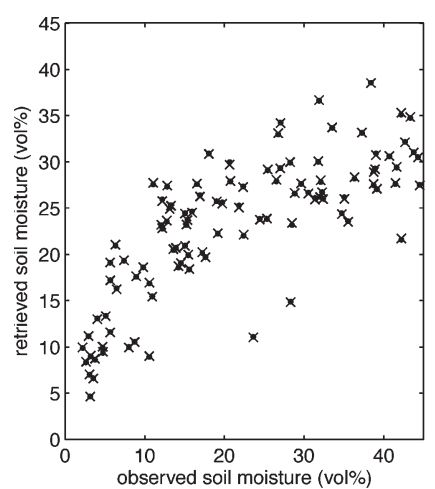

(a)

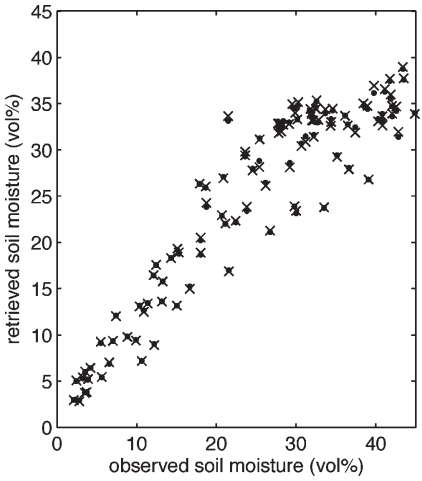

(b)

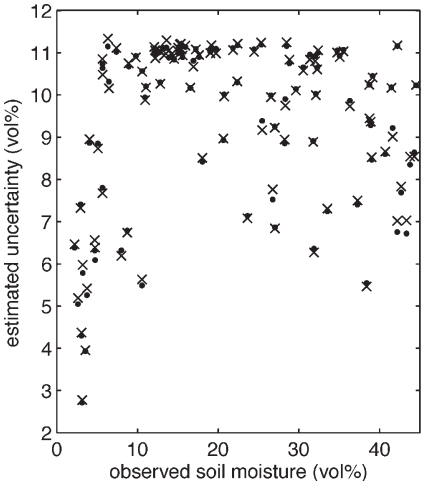

(a)

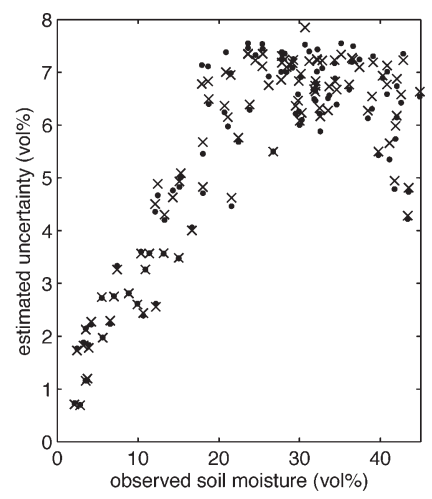

(b)

Fig. 9. Possibilistic retrieved (dots) and Takagi-Sugeno modeled (crosses) soil moisture versus observed soil moisture for (a) roughness class 1 and (b) roughness class 2 .

The objective of identifying the Takagi-Sugeno models for the retrieval of soil moisture and the uncertainty upon the retrieval was to overcome the major disadvantage of the original possibilistic retrieval procedure of the high demand of CPU time. A small experiment, in which the possibilistic and the Takagi-Sugeno alternative were run on 100 data points, showed that both methods retrieve soil moisture with the same accuracy. However, a tremendous gain in runtime is obtained when the

Fig. 10. Possibilistic (dots) and Takagi-Sugeno modeled (crosses) estimated uncertainty versus observed soil moisture for (a) roughness class 1 and (b) roughness class 2 .

fuzzy rule-based model is applied. Therefore, in an operational mode, the Takagi-Sugeno model should be advised as it can invert a complete radar scene in a couple of minutes. However, if the technique should be applied just once to a couple of data points, then the total time necessary for creating the training and validation data set and the identification of the Takagi-Sugeno models cannot be justified, and we advise to use the possibilistic retrieval procedure as proposed by Verhoest et al. [1]. 


\section{REFERENCES}

[1] N. E. C. Verhoest, B. De Baets, F. Mattia, G. Satalino, C. Lucau, and P. Defourny, "A possibilistic approach to soil moisture retrieval from ERS SAR backscattering under soil roughness uncertainty," Water Resour. Res., DOI: 10.1029/2006WR005295, 2007, in press.

[2] M. Callens, N. E. C. Verhoest, and M. W. J. Davidson, "Parameterization of tillage induced single scale soil roughness from 4-m profiles," IEEE Trans. Geosci. Remote Sens., vol. 44, no. 4, pp. 878-888, Apr. 2006.

[3] Y. Oh and Y. C. Kay, "Condition for precise measurement of soil surface roughness," IEEE Trans. Geosci. Remote Sens., vol. 36, no. 2, pp. 691-695, Mar. 1998.

[4] M. W. J. Davidson, T. Le Toan, F. Mattia, G. Satalino, T. Manninen, and M. Borgeaud, "On the characterization of agricultural soil roughness for radar remote sensing studies," IEEE Trans. Geosci. Remote Sens., vol. 38, no. 2, pp. 630-640, Mar. 2000.

[5] F. Mattia, M. W. J. Davidson, T. Le Toan, C. M. F. D'Haese, N. E. C. Verhoest, A. M. Gatti, and M. Borgeaud, "A comparison between soil roughness statistics used in surface scattering models derived from mechanical and laser profilers," IEEE Trans. Geosci. Remote Sens., vol. 41, no. 7 , pp. 1659-1671, Jul. 2003.

[6] G. Satalino, F. Mattia, M. W. J. Davidson, T. Le Toan, G. Pasquariello, and M. Borgeaud, "On current limits of soil moisture retrieval from ERS-SAR data," IEEE Trans. Geosci. Remote Sens., vol. 40, no. 11, pp. 2438-2447, Nov. 2002

[7] A. K. Fung, Z. Li, and K. S. Chen, "Backscattering from a randomly rough dielectric surface," IEEE Trans. Geosci. Remote Sens., vol. 30, no. 2, pp. 356-369, Mar. 1992.

[8] A. K. Fung, Microwave Scattering and Emission Models and Their Applications. Norwood, MA: Artech House, 1994.

[9] F. Mattia and T. Le Toan, "Backscattering properties of multi-scale rough surfaces," J. Electromagn. Waves Appl., vol. 13, no. 4, pp. 491-526, 1999.

[10] F. Mattia, G. Satalino, L. Dente, and G. Pasquariello, "Using a priori information to improve soil moisture retrieval from ENVISAT ASAR AP data in semi-arid regions," IEEE Trans. Geosci. Remote Sens., vol. 44, no. 4, pp. 900-912, Apr. 2006.

[11] D. Dubois and H. M. Prade, Fundamentals of Fuzzy Sets. Norwell, MA: Kluwer, 2000.

[12] L. Zadeh, "Fuzzy sets as a basis for a theory of possibility," Fuzzy Sets Syst., vol. 1, no. 1, pp. 3-28, 1978.

[13] F. T. Ulaby, R. K. Moore, and A. K. Fung, Microwave Remote Sensing, Active and Passive, vol. 2. Norwood, MA: Artech House, 1982.

[14] Y. Oh, K. Sarabandi, and F. T. Ulaby, "An empirical model and an inversion technique for radar scattering from bare soil surfaces," IEEE Trans. Geosci. Remote Sens., vol. 30, no. 2, pp. 370-381, Mar. 1992.

[15] D. Dubois, L. Foulloy, G. Mauris, and H. Prade, "Probability-possibility transformations, triangular fuzzy sets and probabilistic inequalities," Reliab. Comput., vol. 10, no. 4, pp. 273-297, Aug. 2004.

[16] M.-H. Masson and T. Denoeux, "Inferring a possibility distribution from empirical data," Fuzzy Sets Syst., vol. 157, no. 3, pp. 319-340, Feb. 2006.

[17] N. Baghdadi, C. King, A. Chanzy, and P. Wigneron, "An empirical calibration of the integral equation model based on SAR data, soil moisture and surface roughness measurements over bare soil," Int. J. Remote Sens., vol. 23, no. 20, pp. 4325-4340, 2002.

[18] E. Altese, O. Bolognani, M. Mancini, and P. A. Troch, "Retrieving soil moisture over bare soil from ERS-1 synthetic aperture radar data: Sensitivity analysis based on a theoretical surface scattering model and field data," Water Resour. Res., vol. 32, no. 3, pp. 653-661, 1996.

[19] K. S. Chen, S. K. Yen, and W. P. Huang, "A simple model for retrieving bare soil moisture from radar-scattering coefficients," Remote Sens. Environ., vol. 54, no. 2, pp. 121-126, Nov. 1995.

[20] M. C. Dobson, F. T. Ulaby, M. T. Hallikainen, and M. A. El-Rayes, "Microwave dielectric behavior of wet soil-Part II: Dielectric mixing models," IEEE Trans. Geosci. Remote Sens., vol. GRS-23, no. 1, pp. 35-46, Jan. 1985.

[21] D. Dubois and H. Prade, "A class of fuzzy measures based on triangular norms," Int. J. Gen. Syst., vol. 8, no. 1, pp. 43-61, 1982.

[22] P. Smets, "Constructing the pignistic probability function in a context of uncertainty," in Proc. Uncertainty Artif. Intell., M. Henrion, R. D. Schachter, L. N. Kanal, and J. F. Lemmer, Eds., 1989, vol. 5, pp. 29-39.

[23] T. Takagi and M. Sugeno, "Fuzzy identification of systems and its application to modeling and control," IEEE Trans. Syst. Man Cybern., vol. SMC-15, no. 1, pp. 116-131, 1985.

[24] E. H. Mamdani and S. Assilian, "Experiment in linguistic synthesis with a fuzzy logic controller," Int. J. Man-Mach. Stud., vol. 7, no. 1, pp. 1-13, 1975.
[25] D. Gustafson and W. Kessel, "Fuzzy clustering with a fuzzy covariance matrix," in Proc. IEEE CDC, 1979, pp. 761-766.

[26] I. Gath and A. Geva, "Unsupervised optimal fuzzy clustering," IEEE Trans. Pattern Anal. Mach. Intell., vol. 11, no. 7, pp. 773-781, Jul. 1989.

[27] H. Vernieuwe, B. De Baets, and N. E. C. Verhoest, "Comparison of clustering algorithms in the identification of Takagi-Sugeno models: A hydrological case study," Fuzzy Sets Syst., vol. 157, no. 21, pp. 28762896, Nov. 2006.

[28] G. A. Miller, "The magical number seven, plus or minus two: Some limits on our capacity for processing information," Psychol. Rev., vol. 63, no. 2, pp. 81-97, 1956.

[29] J. S. R. Jang, "ANFIS: Adaptive network-based fuzzy inference system," IEEE Trans. Syst., Man Cybern., vol. 23, no. 3, pp. 665-685, May/Jun. 1993.

[30] E. Ruspini, "A new approach to clustering," Inf. Control, vol. 15, no. 1 pp. 22-32, 1969.

[31] M.-Y. Chen and D. A. Linkens, "Rule-base self-generation and simplification for data-driven fuzzy models," Fuzzy Sets Syst., vol. 142, no. 2, pp. 243-265, Mar. 2004.

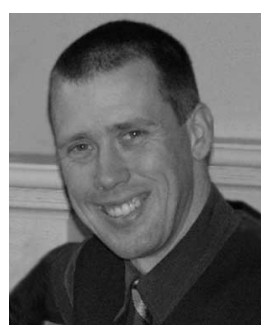

Niko E. C. Verhoest received the engineering and Ph.D. degrees in applied biological sciences from Ghent University, Ghent, Belgium, in 1994 and 2000, respectively.

He was a Teaching Assistant from 1998 to 2000 and an Assistant Professor from 2000 to 2002 with the Laboratory of Hydrology and Water Management, Ghent University. In 2002, he became an Associate Professor of hydrology and water management in the Faculty of Bioscience Engineering, Ghent University. His research interests include hydrological applications of radar remote sensing.

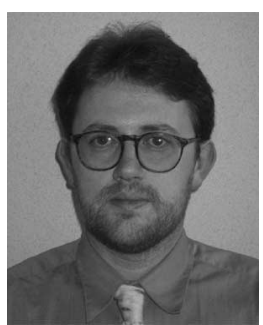

Bernard De Baets was born in 1966. He received the M.Sc. degree in mathematics (summa cum laude), the Postgraduate degree in knowledge technology (summa cum laude), and the Ph.D. degree in mathematics (summa cum laude) from Ghent University, Ghent, Belgium, in 1988, 1991, and 1995, respectively.

Since 1999, he has been an Associate Professor in applied mathematics in the Department of Applied Mathematics, Biometrics and Process Control, Ghent University, where he is leading KERMIT, which is a research unit for knowledge-based systems. He was an Honorary Professor at Budapest Tech in 2006. His publications comprise more than 130 papers in international journals and about 40 book chapters. He coordinates the EURO Working Group on Fuzzy Sets and is a member of the Board of Directors of the European Society for Fuzzy Logic and Technology, the Technical Committee on Artificial Intelligence and Expert Systems of the International Association of Science and Technology for Development, and the Administrative Board of the Belgian OR Society. He serves on the Editorial Boards of various international journals, particularly as Co-Editor-in-Chief of Fuzzy Sets and Systems.

Dr. De Baets was the recipient of the Government of Canada Award in 1988.

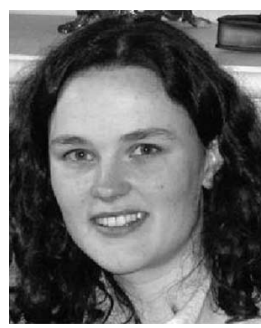

Hilde Vernieuwe was born in 1977. She received the engineering and $\mathrm{Ph} . \mathrm{D}$. degrees in applied biological sciences from Ghent University, Ghent, Belgium, in 2000 and 2005, respectively.

In January 2001, she was with KERMIT, which is the research unit for knowledge-based systems, conducting the Ph.D. degree in the fields of fuzzy modeling and hydrology. Since January 2007, she has been a Postdoctoral Researcher in KERMIT, Department of Applied Mathematics, Biometrics and Process Control, Ghent University. 